\title{
Chronic Inflammation in Hemodialysis
}

This Supplement to Blood Purification reports on contributions by several distinguished speakers who were invited to deal with 'Chronic Inflammation in Hemodialysis' as part of the Permanent Technical Forum at the ERA-EDTA Congress in Madrid, September 1999. In a two-session meeting, several aspects inherent to the biological mechanisms pertinent to the triggering and maintenance of a chronic inflammatory state in ESRD patients on hemodialysis were dealt with. There is consensus over the fact that uremia constitutes the major culprit responsible for the initiation of chronic inflammation. Chronic inflammation has been linked to the development of several medium- to long-term morbidities in ESRD patients on regular extracorporeal treatment.

Cardiovascular disease is the leading cause of mortality in the hemodialysis population. The inflammatory process is instrumental not only to the triggering but also to the persistence of the different factors (activated cells, modified proteins) capable of initiating vascular damage. The role of oxidization in the modification of lipid and protein was discussed by Dr. Ziouzenkova. The recent demonstration of increased levels of the highly pro-atherosclerotic electronegative form of LDL (LDL-) in hemodialysis patients is strongly suggestive of a relevant role of oxidative mechanisms. Beyond the mecha- nisms apparent from hemoincompatibility-related phenomena (elegantly reviewed by Dr. Cristol), a novel implication of hemoglobin (heme)-induced cross-linking with tyrosine residues of LDL was suggested by original results from Dr. Ziouzenkova and her associates and may provide a fresh interest in the often neglected microhemolysis in hemodialysis. Dr. Cristol contributed to our understanding why hemodialysis is a pro-oxidant event. The concomitant diffusive loss of hydrosoluble vitamins (such as vitamin C) may have a greater impact on the oxidative lipid and protein modification than expected so far. Based on recent encouraging data from their respective and independent laboratories, Dr. Cristol and Dr. Ziouzenkova agreed that antioxidant therapies (hemolipodialysis) which combine the effect of scavenging reactive oxygen species and abrogate the loss of vitamin $\mathrm{C}$ deserve attention and future evaluation of their real clinical significance.

A large number of observational studies have provided evidence for the occurrence of hyperhomocysteine and the metabolic pathways involved were exhaustively reviewed by Dr. Schiavon. He discussed the function of homocysteine in chronic renal failure as well as its pharmacokinetics in hemodialysis. Dr. Panichi illustrated the implication of acutephase response and of the 'positive' (C-reactive pro-

\section{KARGER \\ Fax +41613061234 \\ E-Mail karger@karger.ch} www. karger.com (c) 2000 S. Karger AG, Basel

0253-5068/00/0183-0167\$17.50/0

Accessible online at:

www. karger.com/journals/bpu 
tein, serum amyloid A) and of 'negative' (albumin) proteins. He discussed the role of C-reactive protein not only as a marker but also as a mediator of both pro- and anti-inflammatory activities. He also presented very recent data derived from randomized clinical trials and from a cross-sectional survey which suggest the possibility that hemodialysis may reduce the plasma levels of $\mathrm{C}$-reactive protein. $\mathrm{He}$ emphasized the role of backfiltration of contaminated dialysate (within the AAMI standards!) as a chronic stimulus for cytokine production and chronic inflammatory stimulation. The emphasis was put on two possible alternatives, namely the abrogation of backfiltration as it may be feasible in high-volume hemodiafiltration or in double-chamber (paired filtration dialysis) hemodiafiltration, or the use of ultrapure dialysate. Regarding hemodialytic strategies that could reduce the chronic inflammatory state, Dr. De Francisco presented current data on a recently proposed technique which allows regeneration of the ultrafiltrate. This technique consists of the on-line endogenous reinfusion of pure ultrafiltrate after passage in a sorbent cartridge using a paired filtration dialysis type of hemodialyzer. $\mathrm{He}$ described a 1-year multicenter study where a significant reduction in plasma $\beta_{2}$-microglobulin could be observed. He commented that the reduction could be accounted for by the removal rates and could, at least in part, also be due to an improved biocompatibility of the system. A second strategy was presented by Dr. Pizzarelli. In this technique, named paired hemodiafiltration, the infusion fluid was delivered to a first hemofilter, the second hemodialyzer being the actual hemodiafilter. He emphasized that the production of sterile, ultrapure bicarbonate infusion fluid could allow a fluid volume exchange of up to 40 liters/session and his preliminary results showed that there was no cytokine production. Finally, he stressed the possibility in this technique for an online monitoring of the fiber integrity by way of temporary (5 s) inversion of the infusion pump and prompt reading of hemoglobin by a sensitive blood leak detector. The issue of dialysate microbiological purity was strengthened by Dr. Lonnemann who explained why this must be a mandatory issue. Unfortunately, observational studies do show that the quality of water and dialysis fluids may be very far from that recommended by the AAMI standards in many dialysis centers and pinpoint the trend from regulatory bodies to revise these standards to even more restrictive limits. Dr. Canaud, who has a long and worldwide recognized experience of on-line production of dialysate, stressed the concepts of 'aquavigilance' as part of the quality control process that encompasses strict bacteriological monitoring of the water treatment, dialysis monitors and correct handling of the ultrafilter. Failure to follow rules imposed by the strict laws of Microbiology may have disastrous effects that may have been overlooked for a long time. Dr. Cappelli emphasized the risk inherent to the lack of quality control of microbiological quality of the dialysis system. He drew attention to the nature of the biofilm, the mechanisms that underline its formation and the rather inadequate effects of many disinfectants. Dr. Canaud illustrated the mechanisms of transmembrane passage of the bacterial contaminants present in the dialysate such as backfiltration and backdiffusion. Finally, Dr. Lonnemann illustrated the complex mechanism of cytokine production and of the acute response that leads to the enhanced hepatic production of C-reactive protein.

These meetings have endeavored to present for the first time the multifaceted problems related to chronic inflammation in hemodialysis. We can only hope that this Supplement will offer the stimulus for an ever-improving comprehension of the underlying mechanisms and a better definition of the measures that should be adopted on a day-to-day basis in dialysis centers. Finally, we would also like to acknowledge the staff of the Clinical and Laboratory Research Department at Bellco SpA for their scientific and organizational support.

\author{
Julio Botella, Madrid \\ Horst Klinkmann, Rostock \\ Giuseppe La Greca, Vicenza \\ Pietro Zucchelli, Bologna
}

